\title{
Proteasome and its inhibitors from bacteria and fungi
}

\section{Abstract}

The Ubiquitin Proteasomal System is one of the major proteolytic pathways in eukaryotic cells and is required for the regulation and execution of numerous cellular processes. Specifically, it is required for the progression of the cell cycle, in antigen processing, in mounting an inflammatory response as well as for the degradation of damaged and miss-folded proteins. Unregulated Proteasomal activity leads to several human pathological conditions including cancer. The proteasome is thus a target for the development of drugs for the treatment of several human disease conditions. Here we summarize proteasomal inhibitors isolated from bacteria and fungi.

Keywords: Ubiquitin, ubiquitin proteasomal pathway, proteasome, proteasome inhibitors
Volume 2 Issue 3 - 2016

\author{
Ramesh C Meena,Amitabha Chakrabarti \\ Department of Molecular Biology, Defence Institute of \\ Physiology and Allied Sciences, India \\ Correspondence: Amitabha Chakrabarti, Department of \\ Molecular Biology, Defence Institute of Physiology and Allied \\ Sciences, Lucknow Road, Timarpur, Delhi-I 10 054, India, Tel \\ +9| | |23883 |64, Fax +9 I I 23932869, \\ Email achakrabarti_molbiol_dipas@yahoo.co.in
}

Received: June 17, 2016 | Published: July 15, 2016

\section{Introduction}

\section{The Ubiquitin Proteasomal System (UPS)'}

$\mathrm{Ub}$ dependent protein degradation is a complex multistep process. In the first step, ubiquitin activating enzyme (E1) reacts with $\mathrm{Ub}$ in the presence of ATP to yield a ternary complex $\left(\mathrm{E} 1-\mathrm{Ub}_{2}\right)$ in which one $\mathrm{Ub}$ moiety is bound to the enzyme as an Ub-adenylate and the other Ub molecule is bound by a thioester bond between a conserved Cysresidue in E1 and the C-terminal Gly-residue of Ub. Subsequently, the thioester-linked Ub in E1 is transferred to a conserved active-site Cysresidue in one of the many E2 enzymes by a trans-thioesterification reaction. Finally, in the presence of an E3 enzyme, Ub is attached to a substrate protein by an amide isopeptide linkage to a $\varepsilon$-amino group of a Lys-residue in the substrate. Polyubiquitination of substrates is achieved by successive rounds of the above reactions and may require more than one E2 enzyme for optimal activity. A polyubiquitin chain consisting of a minimum of four $\mathrm{Ub}$ moieties $\left(\mathrm{Ub}_{4}\right)$ needs to be linked to proteins to mark them for degradation by the $26 \mathrm{~S}$ proteasome.

The $26 \mathrm{~S}$ proteasome is a proteolytic complex consisting of a hollow cylindrical 20S catalytic core sandwiched between two 19S regulatory subunits. The catalytic core consists of four stacked rings of which the two outer rings are made up of seven different $\alpha$-subunits and the two inner rings containing the catalytic activities are made up of seven different $\beta$ subunits. Substrate entry into the catalytic core is tightly regulated by the $19 \mathrm{~S}$ regulatory particles which constitute the lid of the 20S cylinder. The poly-ubiquitin moiety is recognized and cleaved from the proteasome substrate by the 19S regulatory particle which is subsequently recycled. The 19S subunit also unfolds the proteasome substrate thus aiding in its entry into the $20 \mathrm{~S}$ cylinder for degradation. The catalytic core has six active protease sites, two in subunit $\beta 5$ termed chymotrypsin-like, two in subunits $\beta 2$ termed trypsin-like and the rest in subunit $\beta 1$ termed caspase-like.

\section{The UPS and disease ${ }^{1,2}$}

The UPS is directly related to certain human diseases including Liddle syndrome arising out of inhibition of UPS mediated degradation of epithelial $\mathrm{Na}^{+}$channel; increased proteasomal activity on the other hand has been associated with the pathogenesis of cystic fibrosis, hereditary diabetes, Wilson disease etc. Unregulated UPS mediated degradation of cell cycle regulators e.g., p31 kipl (inhibitor of cyclin dependent kinase) and tumor suppressors e.g., p53 lead to tumorigenesis. Increased degradation of other regulatory molecules including Bax and p105 (NF- $\mathrm{kB}$ precursor) has been shown to be related to inflammatory response and drug resistance in human cancers.

\section{The p53 model}

The p53 tumor suppressor is a critical factor in the determination of cell fate i.e., the decision between proliferation and cell death and is exquisitely regulated at the level of protein stability by the UPS. It is rapidly degraded by the UPS and is stabilized by inhibition of its proteasomal degradation. Once stabilized, it inhibits cell cycle progression. The gene encoding $\mathrm{p} 53$ is mutated in a number of cancers leading to the production of inactive and/or unstable $\mathrm{p} 53$ protein which ultimately leads to tumor formation. Given the above, protesomal inhibitors are thus likely to restore levels of tumor suppressors and are actively sought after in cancer therapeutics.

\section{Proteasome Inhibitors2-4}

Investigation in recent years has yielded a plethora of proteasomal inhibitors; only those of bacterial or fungal origin will be briefly mentioned.

\section{$\beta$-lactones}

A widely used proteasomal inhibitor in research, lactacystin is one of the first proteasomal inhibitors to be isolated and characterized. Isolated from Streptomyces, clasto-lactacystin- $\beta$-lactone formed from lactacystin at neutral $\mathrm{pH}$ has been shown to bind the active site threonine residues in the $\beta$-subunit of the mammalian proteasome. Belactocin C from Streptomyces and Salinosporamide A from (Salinispora tropica) are also members of the $\beta$-lactone group of proteasome inhibitors. 


\section{Epoxyketone}

Epoxomicin from Actinomycetes and Carmaphycin A from Cyanobacterium symploca belong to the epoxyketone group of proteasome inhibitors. Both the compounds specifically inhibit the chymotrypsin like activity of the proteasome and the former has been shown to inhibit NF- $\mathrm{KB}$ activation.

\section{Biaryl compounds}

TMC95A and its diastereomers TMC95B, C and D are proteasomal inhibitors isolated from Apiospora montagnei. All the isomers are able to inhibit the chymotrypsin-like, trypsin-like and caspase like activities of the proteasome with TMC95A being the most potent.

\section{Peptide aldehydes}

Fellutamide B isolated from Penicillum fellutalumis a peptide aldehyde proteasome inhibitor and induces Nerve Growth Factor secretion from fibroblasts and glial-derived cells thereby establishing a relationship between proteasome inhibition and NGF production.

\section{Syrbactins}

Syringolin A from Pseudomonas syringae, Glidobactin (from Photorhabdus) and Cepafungin (from Burkholderiales) are the latest group of proteasomal inhibitors. They all have a twelve-membered macro-lactam ring attached to either a carboxylic acid or a fatty acid tail.

\section{Mechanism of action ${ }^{4}$}

X-ray crystallographic studies of proteasome-inhibitor complexes have indicated that in general most proteasomal inhibitors become covalently attached to the active site threonine residue in the $\beta-5$ subunit. The TMC95 group of compounds however bind noncovalently to all the catalytic subunits and thus inhibits all proteasomal activities.

\section{Biological effects of proteasome inhibitors ${ }^{2}$}

Since the UPS is involved in the regulation and execution of numerous cellular functions it is not surprising that proteasomal inhibitors have wide ranging effects on cell physiology. Protein turnover in general is inhibited by proteasomal inhibitors leading to the intracellular accumulation of ubiquitinated protein which in turn leads to the induction of Heat Shock Proteins (HSPs). Prolonged exposure to proteasome-inhibitors has cytotoxic effects ultimately leading to cell death. In addition, the said compounds show anti-tumor effects which is largely induced by the stabilization of tumor supprssor proteins. Proteasome inhibitors also show anti-inflammatory and immune-suppressive effects.

Proteasomal inhibitors with more specificity and lower side effects are thus the need of the day for the treatment of several human pathologies including a number of cancers.

\section{Acknowledgements}

The authors would like to acknowledge Dr Shashi Bala Singh, OS \& Director DIPAS and Dr Soma Sarkar, Scientist 'F' \& HOD Department of Molecular Biology DIPAS for their support during the course of writing the manuscript.

\section{Conflict of interest}

The author declares no conflict of interest.

\section{References}

1. Schwartz AL, Ciechanover A. The Ubiquitin-proteasome pathway and pathogenesis of human disease. Ann Rev Med. 1999;50:57-74.

2. Kisselev AF, Goldberg AL. Proteasome inhibitors: from research tools to drug candidates. Chem Biol. 2001;8(8):739-758.

3. Delcros JG, Floc h MB, Prigent C, et al. Proteasome Inhibitors as therapeutic agents: current and future strategies. Curr Med Chem. 2003;10(6):479-503.

4. Moore BS, Eustaquio AS, McGlinchey RP. Advances in and applications of proteasome inhibitors. Curr Opin in Chem Biol. 2008;12(4):434-440. 